\title{
The 70-kDa protein bound to hsp90 in wheat germ lysate is a plant homologue of animal Hop
}

\author{
Priti Krishna $^{\mathrm{a}, *}$ and Kimon C. Kanelakis ${ }^{\mathrm{b}}$ \\ ${ }^{a}$ Department of Biology, The University of Western Ontario, London, ON, Canada N6A $5 B 7$ \\ ${ }^{\mathrm{b}}$ Department of Pharmacology, The University of Michigan Medical School, Ann Arbor, MI 48109, USA \\ *Corresponding author, e-mail: pkrishna@uwo.ca
}

Received 20 November 2002; revised 8 April 2003

\begin{abstract}
The hsp90-based chaperone machinery is implicated in numerous cellular processes including signal transduction, genomic silencing, and protein degradation. Hop is a component of the animal hsp90 multichaperone complex, whose function is to link the two chaperones, hsp90 and hsp70. Currently there exists little information on a plant Hop homologue. Herein it is reported that a 70-kDa protein in wheat germ lysate is associated with hsp90 and hsp70 and that this protein is a wheat homologue of Hop. It is also shown that, in addition
\end{abstract}

to being detected in complexes, the wheat Hop as well as the previously identified immunophilin FKBP73, can bind directly to purified plant hsp90. In the steroid receptor folding assay, the wheat Hop was not detected in receptor complexes, but the wheat immunophilin FKBP73 could be detected when mammalian p23 was added to the plant lysate. The present results identify two hsp90-binding proteins and provide a useful framework on which to further investigate their functions.

\section{Introduction}

Several kinases and transcription factors, including the animal steroid hormone receptors (SR), depend on the molecular chaperone hsp90 for acquisition of their functional conformation (Pratt and Toft 1997). The study of hsp90 complexes, in particular those involving animal SRs, has revealed that in addition to hsp90 several other chaperone components participate in the conformational regulation of hsp90 target proteins. The co-chaperones that directly bind to hsp90 include Hop, p23 and the high molecular weight immunophilins (Pratt and Toft 1997). Hop (the hsp70 and hsp90 organizing protein) derives its name from its role in the chaperone machinery as an adapter protein that can bind to both these chaperones simultaneously, bringing them into close proximity (Chen and Smith 1998). This would be significant if, as proposed (Young et al. 2001), hsp70 first contacts the target protein and then facilitates the transfer of the target protein to hsp90. Consistent with such a loading mechanism, Hop and hsp70 dissociate from SR-hsp90 complexes as these complexes progress to the mature form (Chen et al. 1996). The appearance of p23 and a high molecular weight immunophilin in the SR-hsp90 complexes correlates with the final steps of receptor maturation (Johnson et al. 1996). The p23 stabilizes SR-hsp90 complexes in the steroidbinding state (Dittmar et al. 1997), but the function of the immunophilin in this complex is not clear.

Hop binds preferentially to hsp90 and hsp70 in their ADP-bound conformations and the hsp90-Hop-hsp 70 complex is not affected by geldanamycin (GA), a specific inhibitor of hsp90 (Johnson et al. 1998). The binding of Hop to hsp90 is via a central tetratricopeptide repeat (TPR) domain of Hop and to hsp70 via an N-terminus TPR domain (Chen et al. 1996). In addition to its role as an adapter protein, Hop can also regulate hsp90 function by modulating the ATPase activity of hsp90 (Prodromou et al. 1999). Hop homologues have been identified in several organisms (Chen and Smith 1998). With the exception of a soybean heat shock-inducible gene encoding a TPR domain protein that shares amino acid

Abbreviations - GA, geldanamycin; GR, glucocorticoid receptor; HRP, horse radish peroxidase; SR, steroid hormone receptor; TPR, tetratricopeptide repeat; WGL, wheat germ lysate; wHop, wheat Hop. 
identities of 44 and $38 \%$ with the human and yeast Hop homologues, respectively (Torres et al. 1995), no further information on a plant Hop-like protein was available. Earlier, we demonstrated the presence of an hsp90-based chaperone complex in wheat germ lysate (WGL) that can fold the human glucocorticoid receptor (GR) to the hormone-binding state, and identified the presence of wheat hsp90, hsp70, and high molecular weight immunophilins in these complexes (Owens-Grillo et al. 1996, Stancato et al. 1996, Reddy et al. 1998). Recently we have shown that a soybean Hop-like protein binds to hsp90 (Zhang et al. 2003). To further characterize the hsp90 system in WGL, we determined whether a Hop-like protein exists in WGL. Furthermore, since the wheat immunophilin FKBP73 and hsp90 interaction was previously detected in the presence of animal p23 that stabilizes the ATP-bound form of hsp90, we investigated the binding of hsp90 and FKBP73 in the absence of animal $\mathrm{p} 23$. Since a functional assay involving a plant hsp90 target protein is currently not available, we used mammalian GR to assemble a GR-hsp90 complex in WGL to see how interactions of the plant proteins compare with those of their animal counterparts.

\section{Materials and methods}

\section{Materials}

WGL, horse radish peroxidase (HRP)-conjugated antimouse IgG and HRP-conjugated anti-rabbit $\operatorname{IgG}$ were purchased from Promega (Madison, WI, USA). Protein A-Sepharose was from Pharmacia Biotech. (Quebec, Canada), Ni-NTA agarose from Qiagen (Valencia, CA, USA) and mouse IgG1 Kappa (MOPC-21) clarified ascites was from Sigma (St. Louis, MO, USA). ECL Western blotting detection reagents were from Amersham (Piscataway, NJ, USA). The monoclonal antibody DS14F5 (F5) was a generous gift from Dr D.F. Smith (Mayo Clinic Scottsdale, AZ, USA) and was used in the detection and immunoadsorption of a plant Hop homologue. The rabbit $R_{2}$ antiserum (Krishna et al. 1997) was used to detect wheat hsp90, and a polyclonal antiserum kindly provided by $\operatorname{Dr} \mathrm{E}$. Vierling (University of Arizona, Tucson, AZ, USA) was used for the detection of hsp70. FKBP73 was detected using a polyclonal antiserum generously provided by Dr A. Breiman (Tel Aviv University, Tel Aviv, Israel). Purified p23 and GA were kind gifts from Dr W. B. Pratt (University of Michigan, Ann Arbor, MI, USA). The BuGR2 monoclonal antibody against the GR was from Affinity Bioreagents (Golden, CO, USA).

\section{Plant material and growth conditions}

Wheat (Triticum aestivum) seedlings were grown at $23^{\circ} \mathrm{C}$ in a growth chamber under a light intensity of $125 \mu \mathrm{E} \mathrm{m}^{-2} \mathrm{~s}^{-1}$. Leaves and flowers were collected at the appropriate developmental stages and quick-frozen in liquid nitrogen.

\section{Preparation of total cellular proteins}

Frozen plant tissue was ground using a pestle and mortar in extraction buffer $(25 \mathrm{~m} M$ Tris- $\mathrm{HCl}$ pH 8.0, $1 \mathrm{mM}$ EDTA pH 8.0, $20 \mathrm{~m} M$ NaCl, $1 \mathrm{~m} M$ PMSF, $2 \mathrm{~m} M$ benzamidine, $1 \mu \mathrm{g} \mathrm{ml}^{-1}$ leupeptin and $2 \mu \mathrm{g} \mathrm{ml}^{-1}$ aprotinin). The homogenate was centrifuged twice at $12000 \mathrm{~g}$ for $30 \mathrm{~min}$ in an Eppendorf centrifuge at $4^{\circ} \mathrm{C}$. Protein concentration was determined by the Bradford method (Bio-Rad, ON, Canada). Protein samples were aliquoted and an equal volume of $2 \times$ SDS-sample buffer $(0.125 \mathrm{M}$ Tris- $\mathrm{HCl}$ pH 6.8, 4\% SDS, 20\% glycerol, $0.004 \%$ bromophenol blue and $5 \% \quad \beta$-mercaptoethanol) was added prior to storage at $-20^{\circ} \mathrm{C}$.

\section{Immunoadsorption of a Hop homologue from WGL}

The F5 antibody was prebound to protein A-Sepharose by incubating $40 \mu \mathrm{l}$ of a $20 \%$ slurry of protein A-Sepharose with $25 \mu \mathrm{l}$ of ascites in $100 \mu \mathrm{l}$ of HEG buffer $(10 \mathrm{mM}$ HEPES $\mathrm{pH} 7.4,1 \mathrm{mM}$ EDTA, and $10 \%$ glycerol) for $2 \mathrm{~h}$ at $4{ }^{\circ} \mathrm{C}$ with re-suspension of the pellets every 5-10 min The F5-protein A-Sepharose pellets were collected by centrifugation and washed twice with $1 \mathrm{ml}$ of HEG buffer, followed by incubation with $60 \mu \mathrm{l}$ of WGL for $2 \mathrm{~h}$ at $4^{\circ} \mathrm{C}$ with re-suspension of the pellets every $5 \mathrm{~min}$ The non-immune-protein A-Sepharose pellets were prepared similarly and incubated with WGL as described for F5 immune pellets. The non-immune and immune pellets were washed three times by suspension and centrifugation in $1 \mathrm{ml}$ of HEG buffer containing $100 \mathrm{mM} \mathrm{NaCl}$. Immunoadsorbed proteins were extracted in SDS-sample buffer and analysed either by western blotting or silver staining of the gel.

\section{Peptide sequencing}

To obtain the peptide sequences, the gel was both stained with Coomassie Blue and de-stained for short periods of time. The band at $70 \mathrm{kDa}$ was excised and transferred to a microcentrifuge tube, followed by washing twice with $50 \%$ acetonitrile. The gel slice was stored frozen at $-20^{\circ} \mathrm{C}$ and shipped on dry ice for sequencing. The sequence analysis was performed at the Harvard Microchemistry facility (Boston, MA, USA) by microcapillary reverse-phase HPLC nano-electrospray tandem mass spectrometry $(\mu \mathrm{LC} / \mathrm{MS} / \mathrm{MS})$ on a Finnigan LCQ (Thermo Finnigan, San Jose, CA, USA) quadrupole ion trap mass spectrometer.

\section{GR heterocomplex formation}

Expression of GR in $\mathrm{Sf} 9$ cells was as described in Morishima et al. (2000). GR was immunoadsorbed from Sf9 cell lysate by incubating $50 \mu$ lof protein A-Sepharose pellets with $10 \mu \mathrm{l}$ of BuGR2 antibody, $50 \mu 1$ of Sf9 cell lysate and $125 \mu \mathrm{l}$ of TEG buffer $(10 \mathrm{mM}$ TES, $50 \mathrm{mM}$ $\mathrm{NaCl}, 1 \mathrm{~m} M$ EDTA, and $10 \%$ glycerol, $\mathrm{pH}$ 7.6) for $2 \mathrm{~h}$ at $4{ }^{\circ} \mathrm{C}$ with re-suspension of the pellets every $5-10 \mathrm{~min}$. The immunoadsorbed receptors were stripped of associated 
proteins by addition of $\mathrm{NaCl}$ to $0.6 \mathrm{M}$ and incubation of the immune pellet for $2 \mathrm{~h}$ at $4{ }^{\circ} \mathrm{C}$, followed by one wash with $1 \mathrm{ml}$ of TEG buffer and a second wash with $10 \mathrm{mM}$ Hepes, pH 7.5. The immune pellets were next incubated with $70 \mu \mathrm{l}$ of $\mathrm{WGL}$ for $30 \mathrm{~min}$ at $30^{\circ} \mathrm{C}$ with frequent re-suspension, followed by three washes with $1 \mathrm{ml}$ of TEG buffer. Immunoadsorbed proteins were extracted in SDS-sample buffer and analysed by western blotting.

\section{Western blotting}

To identify a Hop homologue in plants, $100 \mu \mathrm{g}$ of total protein from each sample was separated on a 7.5\% SDSpolyacrylamide gel and transferred to nitrocellulose membranes by electroblotting using the Trans-Blot Semi-Dry Electrophoretic Transfer Cell (Bio-Rad). The wheat Hop-like protein (wHop) was detected by sequential incubation with the F5 anti-body at a dilution of $1: 1000$ and HRP-conjugated antimouse IgG at a dilution of $1: 5000$, followed by enhanced chemiluminescence (ECL System; Amersham).

For identification of immunoadsorbed and co-immunoadsorbed proteins, the protein A sepharose pellets following all incubations and washes were heated in SDS sample buffer. The released proteins were resolved on $7.5 \%$ SDS-polyacrylamide gels and transferred to nitrocellulose membranes. The membranes were probed with either the $\mathrm{R}_{2}$ (anti-hsp90) and anti-hsp70 antibodies each at a dilution of $1: 5000$ or F5 antibody at a dilution of $1: 1000$ followed by incubation with the appropriate HRP-conjugated secondary antibody. The GR was probed with the BuGR2 antibody at a dilution of $1: 500$ and the FKBP73 was detected with the antiFKBP73 antibody at a dilution of $1: 10000$.

\section{Expression and purification of a recombinant Brassica napus hsp90 from bacterial cells}

A $5^{\prime}$ end primer containing the BamHI restriction site and codons for initiating methionine and six histidine residues was used in combination with a $3^{\prime}$ end primer to modify a full-length B.napus hsp90 cDNA clone (Krishna et al. 1995) by PCR in order to generate $\mathrm{N}$-terminal his-tagged hsp90. The PCR product was first cloned into the TA cloning vector (Invitrogen, Carlsbad, CA, USA) and its sequence was verified. The insert was lifted out of the TA cloning vector by digestion with BamHI (the second BamHI site was in the TA cloning vector) and ligated into the BamHI site of the pET-11a (Novagen, Madison, WI, USA) expression vector. The pET-11a hsp90 expression construct was transformed into Escherichia coli BL21 cells.

For expression of his-tagged hsp90, BL21 cells carrying the expression construct were grown to an $\mathrm{OD}_{600}$ of 0.8 , induced at $30^{\circ} \mathrm{C}$ by addition of $1 \mathrm{~m} M$ IPTG and then harvested by centrifugation after $2 \mathrm{~h}$. The cells were washed with $20 \mathrm{~m} M$ Hepes pH 7.4, $1 \mathrm{~m} M$ EDTA, disrupted by sonication in buffer A (20 $\mathrm{m} M$ Hepes $\mathrm{pH}$ 8.0, $20 \mathrm{~m} M \mathrm{NaCl}, 1 \mathrm{~m} M$ DTT, $1 \mathrm{~m} M$ PMSF, $1 \mathrm{~m} M$ benzamidine, $1 \mu \mathrm{g} \mathrm{ml}^{-1}$ leupeptin, $2 \mu \mathrm{g} \mathrm{ml}^{-1}$ aprotinin, $10 \mathrm{~m} M$ imidazole and $0.1 \% \mathrm{NP}-40$ ) and lysates were recovered by centrifugation. His-tagged hsp90 was purified by chromatography on Ni-NTA agarose, which was equilibrated in buffer A. Following extensive washing with buffer A, the bound protein was eluted in buffer B (10 $\mathrm{m} M$ Hepes pH 8.0, $20 \mathrm{~m} M \mathrm{NaCl}, 1 \mathrm{~m} M$ DTT and $250 \mathrm{~m} M$ imidazole), dialysed against HEG buffer and stored in aliquots at $-80^{\circ} \mathrm{C}$ until required. The protein was assessed for purity by SDS-polyacrylamide gel electrophoresis and Coomassie staining.

Ni-NTA agarose $(50 \mu \mathrm{l}$ of a slurry), equilibrated in buffer C (20 mM Hepes pH 8.0, $20 \mathrm{~m} M \mathrm{NaCl}$, and $1 \mathrm{~m} M$ DTT), was incubated with $15 \mu \mathrm{g}$ of purified histagged hsp90 for $30 \mathrm{~min}$ at $4^{\circ} \mathrm{C}$ with re-suspension every $5 \mathrm{~min}$. The beads were washed twice with buffer $\mathrm{C}$ and then incubated with $35 \mu \mathrm{l}$ of WGL for $30 \mathrm{~min}$ at $4^{\circ} \mathrm{C}$ with re-suspension every $5 \mathrm{~min}$. Following three washes with buffer $\mathrm{C}$, the proteins were extracted in SDS-sample buffer and analysed by Coomassie staining and western blotting.

\section{Results}

The monoclonal antibody DS14F5, referred to as F5, was raised against purified chicken Hop (Smith et al. 1993) and has been used extensively in the study of Hop complexes in animal cell lysates (Chen et al. 1996, Chen and Smith 1998). We used this antibody in a western blot assay to investigate the presence of a similar protein in WGL and in wheat leaves and flowers. The first and second leaves were collected from wheat seedlings, whereas the leaf just below the flower (upper first), the leaf below the upper first (upper second) and the flowers were collected from mature wheat plants. A $70-\mathrm{kDa}$ protein was specifically detected in the wheat organs examined (Fig. 1). Hop is known to bind hsp90 and hsp70 both simultaneously and individually. To gather further evidence in support of the wheat $70-\mathrm{kDa}$ protein as a putative Hop homologue, the F5 antibody bound to protein A-Sepharose was used to immunopurify the wheat $70-\mathrm{kDa}$ protein from WGL under low-salt conditions. Not only could the $70-\mathrm{kDa}$ protein be isolated in the immunoprecipitation experiment, but wheat hsp 70 and hsp90 were also present in the complex, each detected by using a specific antibody to the protein

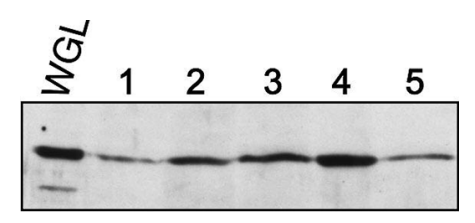

Fig. 1. A Hop homologue is present in wheat. WGL $(2 \mu \mathrm{l})$ and total proteins $(100 \mu \mathrm{g})$ from the first leaf (lane 1), second leaf (lane 2), upper first leaf (lane 3), flower (lane 4), and upper second leaf (lane 5 ) of wheat plants were separated on a $7.5 \%$ SDS-polyacrylamide gel and probed with the F5 antibody following transfer to nitrocellulose. 

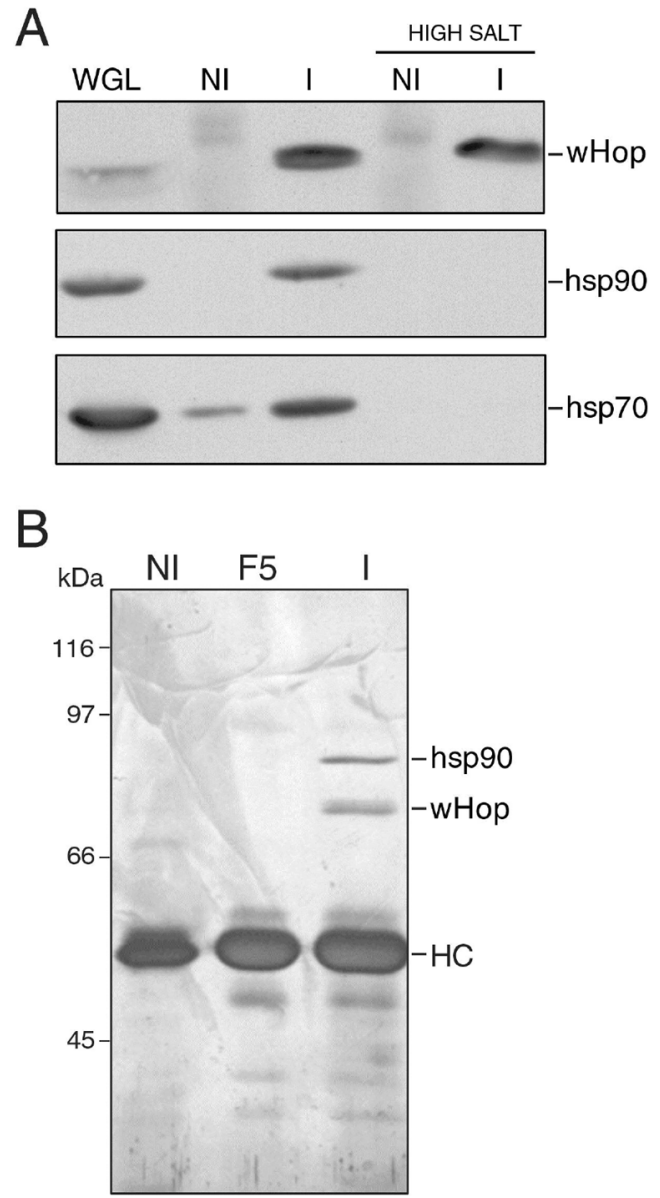

Fig. 2. Immunoprecipitation of a wheat Hop homologue from WGL. A. Protein A-Sepharose pellets coated with either nonimmune IgG (NI) or F5 antibody (I) were incubated with WGL. Pellets were washed with buffer containing either low salt $(0.1 \mathrm{M}$ $\mathrm{NaCl})$ or high salt $(0.5 \mathrm{M} \mathrm{NaCl})$ and proteins were extracted in SDS sample buffer. The extracted proteins were separated on a $7.5 \%$ SDS-polyacrylamide gel and transferred to a nitrocellulose membrane by electroblotting. Wheat Hop, hsp90 and hsp70 were detected using F5, $\mathrm{R}_{2}$ and anti-hsp70 antibodies, respectively. An aliquot of WGL was run on the gel to mark the positions of different proteins. B. Protein A-Sepharose pellets coated with either non-immune IgG (NI) or F5 (I) were incubated with WGL. An equal volume of Protein A-Sepharose pellets coated with F5 were incubated with buffer in place of WGL (F5). Following low-salt washes of the pellets, proteins were extracted in SDS sample buffer and separated on a $7.5 \%$ SDS-polyacrylamide gel. Proteins were visualized by silver staining of the gel. The numbers on the left indicate molecular mass markers in $\mathrm{kDa}$. The band corresponding to the antibody heavy chain (HC) is indicated on the right.

(Fig. 2A, lane I). When the immune pellet was washed with buffer containing $0.5 \mathrm{M} \mathrm{NaCl}$, both hsp90 and hsp70 were dissociated from the complex, but the $70-\mathrm{kDa}$ protein was retained as would be expected if it was bound to the F5 antibody. In some repetitions of the experiment it was noted that hsp70 was reduced, but not completely eliminated from the complex after the highsalt wash. It is known that hsp70 binds non-specifically to protein A-Sepharose beads. Some hsp70 can also be seen in the non-immune pellet due to non-specific binding (Fig. 2A, lane NI).

The 70-kDa protein ran in close proximity to hsp70 on the SDS-polyacrylamide gel as silver staining of proteins showed the presence of a doublet at that position on the gel (Fig. 2B, lane I). One other band was detected at $83 \mathrm{kDa}$, which represents plant hsp90 (Krishna et al. 1995, 1997). Neither band was seen in the non-immune (lane NI) or the antibody alone (lane F5) control. To identify the $70-\mathrm{kDa}$ protein, the doublet was excised after staining the gel with Coomassie blue and shipped to the Harvard Microchemistry facility. Several peptides showing amino acid sequence identity to the hsp70 class of proteins (not shown), and three peptides showing amino acid sequence identity to the previously isolated soybean TPR domain protein (Fig. 3) were identified after $\mu \mathrm{LC} /$ MS/MS analysis. Thus, considering all information, such as the size of the protein recognized by F5, its interaction with hsp90 and hsp70, and the amino acid sequences of internal peptides, we conclude that the $70-\mathrm{kDa}$ protein is a wheat homologue of animal Hop, referred hereafter as wHop.

To further examine the interaction between wHop and hsp90, the F5 antibody bound to protein A-Sepharose was incubated with WGL and the resulting immunoprecipitate was subjected to washes with increasing ionic strength buffer. Figure 4A shows that the interaction between the two proteins was disrupted by $0.3 \mathrm{M} \mathrm{NaCl}$, and completely abolished in the presence of $0.5 \mathrm{M} \mathrm{NaCl}$. We investigated whether wHop stripped of hsp90 by a high-salt wash could be reconstituted with hsp90 following removal of salt and re-incubation with WGL. Hsp90 was eliminated from the complex by washing with $0.5 \mathrm{M}$ $\mathrm{NaCl}$ (Fig. 4B, lane 2) and could be reconstituted with wHop by re-incubation with WGL (lane 3). These results indicate that the interaction between the two proteins is salt sensitive and can be reversed following removal of salt.

During cell free assembly of SRs with hsp90, following an early interaction with hsp70, the SRs are recovered in an intermediate complex with hsp70, Hop and hsp90 and finally in mature complexes containing p23 and one of the three high molecular weight immunophilins (Pratt and Toft 1997). In the presence of GA, this assembly is arrested at an intermediate step resulting in enhanced recovery of SR complexes with hsp90, Hop and hsp70 (Smith et al. 1995). We have previously demonstrated that wheat hsp90, wheat immunophilin FKBP73 and mammalian p23 can be isolated as a complex when immunoadsorbed p23 is incubated with WGL, and that this complex can be disrupted by GA in a temperaturedependent manner (Reddy et al. 1998). It should be noted that because WGL appears to lack a p23-like stabilizing activity, purified mammalian p23 has been used to form stable hsp90 complexes in WGL (Hutchison et al. 1995, Reddy et al. 1998). We wished to study GR assembly in WGL by characterizing the components of GR heterocomplexes formed either in the presence of mammalian p23 added to WGL or in 
2. AAVYLEMGK

\section{HDPNNQELLDGIR}

MAEEAKAKGNAAFSAGDFAAAVRHFSDAIALSPSNHVLYSNRSAATLPPELRGGPSRRQKTVDLKPDWPK AYSRLGAAHLGLRRHRDASPPTKPASNSNPDNAALKSGLADAQAAASRPPPTSPFATAFSGPDMWARSPP TPPHVQPPGPRVRQDHAGHPEGPQQVQPAFEYQRVMHAI GVLLNVKIQTPNHDENDHDADDDVSEDEVVS QPEPEHEPEAAVEVAEEEEEEEKETRDRKGQAQKEKEAGNAAYKKKDFETAIGHYSKALELDDEDISYLT NR/AAVYLEMGKFEDCI KDCEKAVERGKELRSDYKMI ARALTRKGTALAKMAKCSKDFEPAIEIFQKALT ENRNPDTLKKLNEAEKAKKELEQQEYFDPKLADEAREKGNELFKQQKYPEATKHYTEAI KRNPKDAKAYS NRAACYTKLGAMPEGLKDAEKCI ELDPTF SKGYTRKGAVQFSMKEYDKALETYREGLKHDPNNOELLDGI RRCVEQINKASRGDFTPEELKERQAKAMQDPEIQS ILQDPVMTQVLTDFQENPRAAEEHVKNPMVMNKTQ NVTVPGCQMR

Fig. 3. Amino acid sequences of internal peptides of wHop. Protein A-Sepharose pellets coated with F5 antibody were incubated with WGL. After washing the pellet, proteins were extracted in SDS sample buffer, separated on a $7.5 \%$ SDS-polyacrylamide gel and stained with Coomassie blue. The protein band at approximately $70 \mathrm{kDa}$ was excised and sent to the Harvard Microchemistry Facility for sequencing. Three peptides found to share sequence identity with portions of the amino acid sequence of the soybean GMSTI are shown above. Regions within the GMSTI sequence (accession number X79770) corresponding to the three peptides are represented in bold.

the presence of both mammalian p23 and GA added to WGL. Both hsp90 and FKBP73 were detected at low levels in GR complexes that were assembled by incubating immunoadsorbed receptor with WGL (Fig. 5, lane 3). Some hsp90 and FKBP73 was also detected in the nonimmune pellet (lane 1), presumably due to a low level of GR being pulled down in the reaction. In the presence of mammalian p23, the amount of hsp90 and FKBP73 in GR complexes increased significantly (lane 4; compare lanes 3 and 4), indicating that the plant immunophilin is recruited into the GR complex similar to its animal counterpart. Furthermore, this recruitment into the GR complex was inhibited in the presence of GA (lane 5). However, contrary to what is seen when cell free

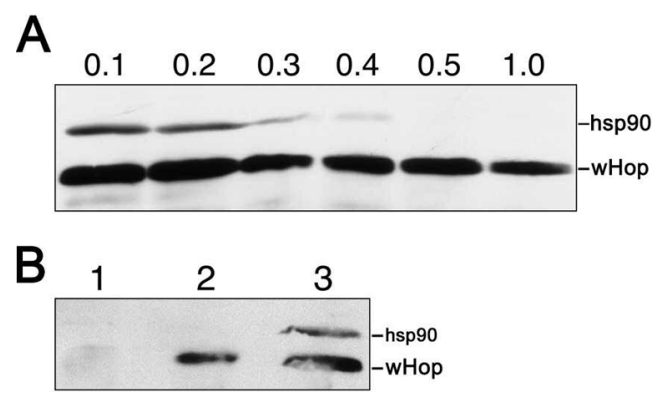

Fig. 4. Hsp90 bound to wHop can be washed away with high salt and reconstituted with it. A. F5-Protein A-Sepharose pellets were incubated with WGL and then washed with HEG buffer containing either $0.1,0.2,0.3,0.4,0.5$ or $1 M \mathrm{NaCl}$. B. Protein A-Sepharose pellets coated with either non-immune IgG or F5 antibody were incubated with WGL. The pellets were washed with HEG buffer containing $0.5 \mathrm{M} \mathrm{NaCl}$ to strip associated proteins from wHop. Following three low-salt washes, the pellets were re-incubated with $60 \mu \mathrm{l}$ of fresh WGL. The pellets were washed with low-salt buffer and proteins were extracted in SDS sample buffer. Extracted proteins were separated on a $7.5 \%$ SDS-polyacrylamide gel and transferred to a nitrocellulose membrane. Hsp90 and the wHop were detected using $\mathrm{R}_{2}$ and F5 antibodies, respectively. Nonimmune IgG-Protein A-Sepharose pellets incubated with WGL, washed with high-salt buffer and re-incubated with WGL (lane 1); F5-Protein A-Sepharose pellets incubated with WGL and washed with high-salt buffer (lane 2); F5-Protein A-Sepharose pellets incubated with WGL, washed with high-salt buffer and re-incubated with WGL (lane 3). assembly of GR is performed with mammalian proteins, wHop was not detected in the presence of GA (lane 5). In lane 4, an additional band between hsp90 and FKBP73 was also detected. A same size band is often detected in WGL due to cross-reactivity of the anti-FKBP73 antibody with a protein coinciding in size with FKBP77, another immunophilin of wheat that also interacts with hsp90 (Reddy et al. 1998). In lane 4, the intensity of the signal is particularly strong presumably due to the recruitment of FKBP77 in GR complexes. Further studies are required to confirm the identity of this protein.

Although we have found that wheat hsp90 and FKBP73 can exist in a complex in the presence of animal p23 (Reddy et al. 1998), it has not as yet been demonstrated that wheat hsp90 and FKBP73 can interact with one another in the absence of mammalian p23. To determine the interaction of plant hsp90 with wheat FKBP73 and with wheat Hop in the absence of any animal protein, a recombinant $B$. napus hsp90 fused to six histidines was expressed and isolated from bacterial cells. The

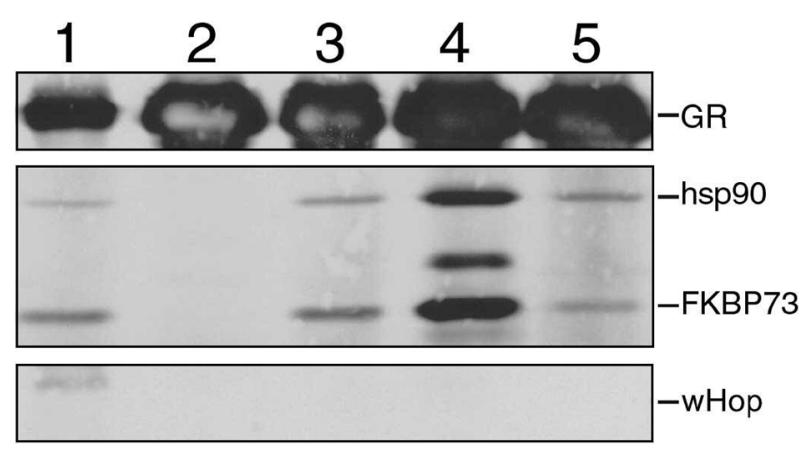

Fig. 5. The wHop is not detected in GR-hsp90 heterocomplexes. Immunoadsorbed stripped GR (immune pellet) was incubated with WGL or WGL plus human p23 $(5 \mu \mathrm{g})$ in the presence or absence of geldanamycin $\left(20 \mu \mathrm{g} \mathrm{ml}^{-1}\right)$. Samples were immunoblotted for GR and associated proteins. Non-immune pellet incubated with WGL (lane 1), stripped GR (lane 2), immune pellet incubated with WGL (lane 3), immune pellet incubated with WGL plus human p23 (lane 4) and immune pellet incubated with WGL plus p23 and geldanamycin (lane 5). 
purified hsp90 was immobilized on Ni-NTA agarose and incubated with WGL. To distinguish between nonspecific interaction of proteins with Ni-NTA agarose and specific interactions with hsp90, the Ni-NTA agarose beads were incubated with WGL as a control (Fig.6, lane 1). Following low-salt washes, the proteins were extracted in SDS sample buffer and analysed by gel electrophoresis and immunoblotting. Both FKBP73 and wHop were retained on the hsp90-Ni-NTA agarose bead complex (lane 2). The wHop showed no non-specific interaction with the Ni-NTA agarose beads, but a low level of FKBP73 was retained on these beads in the absence of hsp90 (lane 1). These results further confirm that the interaction between hsp90 and wHop is authentic and occurs even when hsp90 is from a different plant species and bacterially expressed. These results also demonstrate that hsp90 and FKBP73 interact with one another in the absence of any mammalian p23 added to WGL. The retention of FKBP73 and wHop on hsp90-NiNTA agarose beads does not necessarily indicate a threeway complex between these proteins; such a complex is unlikely based on studies in animal systems, which indicate that immunophilins and Hop compete for a common site on hsp90 (Pratt and Toft 1997).

\section{Discussion}

Over the last decade the hsp90-based chaperone machinery has become implicated in numerous cellular processes including protein folding (Frydman 2001), protein trafficking (Pratt et al. 2001), genomic silencing (Queitsch et al. 2002), and protein degradation (McClellan and Frydman 2001). Hop and hsp70 are part of this multiprotein chaperone machinery. The hsp90-based chaperone system has been identified in plants, but

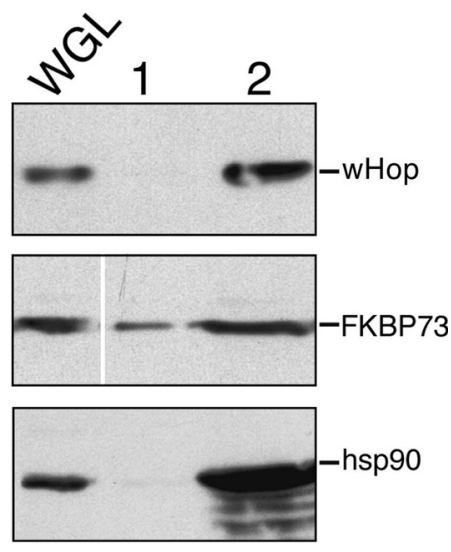

Fig. 6. Recombinant plant hsp90 expressed and purified from bacteria binds to both wHop and FKBP73. His-tagged hsp90 bound to Ni-NTA agarose was incubated with WGL. Following three washes, proteins were extracted in SDS sample buffer, separated on a $10 \%$ SDS-polyacrylamide gel and immunoblotted for wHop, FKBP73 and hsp90. WGL was loaded as a reference for detected proteins. Ni-NTA agarose incubated with WGL (lane 1); his-tagged hsp90 bound to Ni-NTA agarose incubated with WGL (lane 2). awaits further biochemical and molecular characterization. To date hsp70 and the immunophilins have been identified in plant hsp90 complexes (reviewed in Krishna and Gloor 2001). More recently we have described a Hop homologue in soybean that can interact with hsp90 (Zhang et al. 2003). Here we provide the first biochemical evidence for a Hop-like protein in WGL using the F5 antibody raised against avian Hop (Fig. 1). An examination of the Arabidopsis thaliana genome for Hop sequences indicates that at least three Hop orthologues are present in this model plant (Krishna and Gloor 2001). Furthermore, a search of EST databases reveals that transcripts encoding Hop-like proteins are present in different plant species, including wheat. Together these results suggest that the distribution of a Hop homologue is ubiquitous across higher plants and the protein may thus be important for plant cellular functions.

We utilized the model of GR heterocomplex assembly, the best-studied system for delineating the function of the hsp90-Hop-hsp70 complex in animals (Pratt and Toft 1997), to gain insight into the mode of action of the plant complex. Since Hop is found in intermediate SR complexes, we examined both mature and intermediate GR heterocomplexes formed by incubation of the GR with WGL. It is interesting that wHop was neither found in intermediate complexes formed by the addition of GA, nor in mature heterocomplexes (Fig. 5). In contrast, the high-molecular weight immunophilin FKBP73 was detected in these complexes, in particular when stabilized by the addition of human p23 (Fig. 5). There may be a number of reasons why wHop was not detected in GR heterocomplexes. Johnson et al. (1998) have shown that Hop binds preferentially to the ADP-bound form of hsp70 and hsp90. In animal systems, BAG-1, a co-chaperone of hsp70, allows for the exit of Hop from intermediate GR heterocomplexes in vitro (Kanelakis et al. 1999). It is possible that a co-chaperone of either hsp70 or hsp90 in WGL accelerates the conversion of hsp70 and/or hsp90 to the ATP-bound form, the low affinity binding state of Hop, allowing for fast exit of wHop from the GR heterocomplex. We have previously shown that the rate of disassembly of GR-hsp90 heterocomplexes is higher in plant lysates as compared to animal lysates (Stancato et al. 1996). A transient association of wHop with the GR heterocomplex may not be detectable in our system. A less likely, but possible scenario is that the F5 antibody recognizes a specific wHop isoform that does not participate in GR heterocomplex assembly in WGL. In the future, a functional assay involving a plant hsp90 client protein, as well as further characterization of the Hop protein family in plants, will better define the mode of plant Hop action.

We have previously shown that the wheat immunophilin FKBP73 associates with plant hsp90 via its TPR domains in the presence of mammalian p23 (Reddy et al. 1998). Due to the lack of efficient immunoadsorbing antibodies against plant hsp90 and FKBP73, a direct interaction between hsp90 and FKBP73 could not be demonstrated at that time. Using immobilized hsp90 on 
Ni-NTA agarose beads, it is demonstrated here that wheat FKBP73 can interact with B.napus hsp90 in the absence of mammalian p23 (Fig. 6). Not only is the interaction of hsp90 with immunophilins conserved in plants and animals, but also conserved is the recruitment of both proteins into GR heterocomplexes (Fig. 5). The abundance of FKBP73 in GR heterocomplexes was increased in the presence of mammalian p23 (Fig.5). The lack of a p23-like activity in WGL, but the identification of a gene encoding a p23-like protein in A.thaliana (Krishna and Gloor 2001) is enigmatic at present. The study of plant p23 in the future will, no doubt, help in providing a clearer picture of the hsp90 heterocomplexes in plant cells.

In summary, this is the first demonstration that a Hop homologue in WGL can be immunoprecipitated in a complex with hsp90 and hsp70. It can be extrapolated from observations in animal systems that the plant protein functions similarly as an adapter bringing together plant hsp70 and hsp90 into the same multiprotein chaperone complex. However, this remains to be confirmed. While the functions of both the plant Hop homologue and the immunophilins will be best understood when target proteins of plant hsp90 have been identified, the present study identifies two hsp90-binding proteins and lays the foundation on the basis of which further studies can be conducted to understand how the various proteins of the hsp90 multichaperone complex function interactively. Hsp90 heterocomplexes in plants appear to have unique features, and specific knowledge of them is required to understand how proteins involved in signal transduction pathways and other processes are stabilized, functionally modulated and trafficked within plant cells.

Acknowledgements - We would like to thank Dr D.F. Smith for providing the F5 antibody; Dr E. Vierling for the antibody to plant hsp70; Dr A. Breiman for the antibody to FKBP73; and Dr W.B. Pratt for purified p23 and geldanamycin. We thank Professor Perry for helpful suggestions on the manuscript. Technical assistance provided by L. Yue is acknowledged. This work was supported by a research grant to P.K. from the Natural Sciences and Engineering Research Council of Canada.

\section{References}

Chen S, Prapapanich V, Rimerman RA, Honoré B, Smith DF (1996) Interactions of $\mathrm{p} 60$, a mediator of progesterone receptor assembly, with heat shock proteins Hsp90 and Hsp70. Mol Endocrinol 10: 682-693

Chen S, Smith DF (1998) Hop as an adaptor in the heat shock protein 70 (Hsp70) and Hsp90 chaperone machinery. J Biol Chem 273: 35194-35200

Dittmar KD, Demady DR, Stancato LF, Krishna P, Pratt WB (1997) Folding of the glucocorticoid receptor by the heat shock protein (hsp) 90-based chaperone machinery. The role of p23 is to stabilize receptor.hsp90 heterocomplexes formed by hsp90.p60.hsp70. J Biol Chem 273: 7358-7366

Frydman J (2001) Folding of newly translated proteins in vivo: the role of molecular chaperones. Annu Rev Biochem 70: 603-647

Hutchison KA, Stancato LF, Owens-Grillo JK, Johnson JL, Krishna P, Toft DO, Pratt WB (1995) The 23-kDa acidic pro- tein in reticulocyte lysate is the weakly bound component of the hsp foldosome that is required for assembly of the glucocorticoid receptor into a functional heterocomplex with hsp90. J Biol Chem 270: 18841-18847

Johnson J, Corbisier R, Stensgard B, Toft DO (1996) The involvement of p23, hsp90, and immunophilins in the assembly of progesterone receptor complexes. J Steroid Biochem Mol Biol 56: $31-37$

Johnson BD, Schumacher RJ, Ross ED, Toft DO (1998) Hop modulates hsp70/hsp90 interactions in protein folding. J Biol Chem 273: 3679-3686

Kanelakis KC, Morishima Y, Dittmar KD, Galigniana MD, Takayama S, Reed JC, Pratt WB (1999) Differential effects of the hsp70-binding protein BAG-1 on glucocorticoid receptor folding by the hsp90-based chaperone machinery. J Biol Chem 274: 34134-34140

Krishna P, Gloor G (2001) The Hsp90 family of proteins in Arabidopsis thaliana. Cell Stress Chaperones 6: 238-246

Krishna P, Reddy RK, Sacco M, Frappier JR, Felsheim RF (1997) Analysis of the native forms of the $90 \mathrm{kDa}$ heat shock protein (hsp90) in plant cytosolic extracts. Plant Mol Biol 33: 457-466

Krishna P, Sacco M, Cherutti JF, Hill S (1995) Cold-induced accumulation of hsp90 transcripts in Brassica napus. Plant Physiol 107: 915-923

McClellan AJ, Frydman J (2001) Molecular chaperones and the art of recognizing a lost cause. Nature Cell Biol 3: E51-E53

Morishima Y, Kanelakis KC, Silverstein AM, Dittmar KD, Estrada L, Pratt WB (2000) The hsp organizer protein Hop enhances the rate of but is not essential for glucocorticoid receptor folding by the multiprotein hsp90-based chaperone system. J Biol Chem 275: 6894-6900

Owens-Grillo JK, Stancato LF, Hoffmann K, Pratt WB, Krishna P (1996) Binding of immunophilins to the $90 \mathrm{kDa}$ heat shock protein (hsp90) via a tetratricopeptide repeat domain is a conserved protein interaction in plants. Biochemistry 35: $15249-15255$

Pratt WB, Krishna P, Olsen LJ (2001) Hsp90-binding immunophilins in plants: the protein movers. Trends Plant Sci 6: 54-58

Pratt WB, Toft DO (1997) Steroid receptor interactions with heat shock protein and immunophilin chaperones. Endocri Rev 18: 306-360

Prodromou C, Siligardi G, O'Brien R, Woolfson D, Regan L, Panaretou B, Ladbury J, Piper P, Pearl L (1999) Regulation of Hsp90 ATPase activity by tetratricopeptide repeat domain co-chaperones. EMBO J 18: 754-762

Queitsch C, Sangster TA, Lindquist S (2002) Hsp90 as a capacitor for phenotypic variation. Nature 417: 618-624

Reddy RK, Kurek I, Silverstein AM, Chinkers M, Breiman A, Krishna P (1998) High molecular weight FK506-binding proteins are components of heat shock protein 90 heterocomplexes in wheat germ lysate. Plant Physiol 118: 1395-1401

Smith DF, Sullivan WP, Marion TN, Zaitsu K, Madden B, McCormick DJ, Toft DO (1993) Identification of a 60kilodalton stress-related protein, p60, which interacts with hsp90 and hsp70. Mol Cell Biol 13: 869-876

Smith DF, Whitesell L, Nair SC, Chen S, Prapapanich V, Rimerman RA (1995) Progesterone receptor structure and function altered by geldanamycin, an hsp90-binding agent. Mol Cell Biol 15: 6804-6812

Stancato LF, Hutchison KA, Krishna P, Pratt WB (1996) Animal and plant cell lysates share a conserved chaperone system that assembles the glucocorticoid receptor into a functional heterocomplex with hsp90. Biochemistry 35: 554-561

Torres JH, Chantellard P, Stutz E (1995) Isolation and characterization of gmsti, a stress-inducible gene from soybean (Glycine max) coding for a protein belonging to the TPR (tetratricopeptide repeats) family. Plant Mol Biol 27: 1221-1226

Young JC, Moarefi I, Hartl FU (2001) Hsp90: a specialized but essential protein-folding tool. J Cell Biol 154: 267-273

Zhang Z, Quick MK, Kanelakis KC, Gijzen M, Krishna P (2003) Characterization of a plant homolog of Hop, a co-chaperone of hsp90. Plant Physiol 131: 525-535 